\title{
Ginkgo history told by genomes
}

Nat. Commun. 10, 4201 (2019).

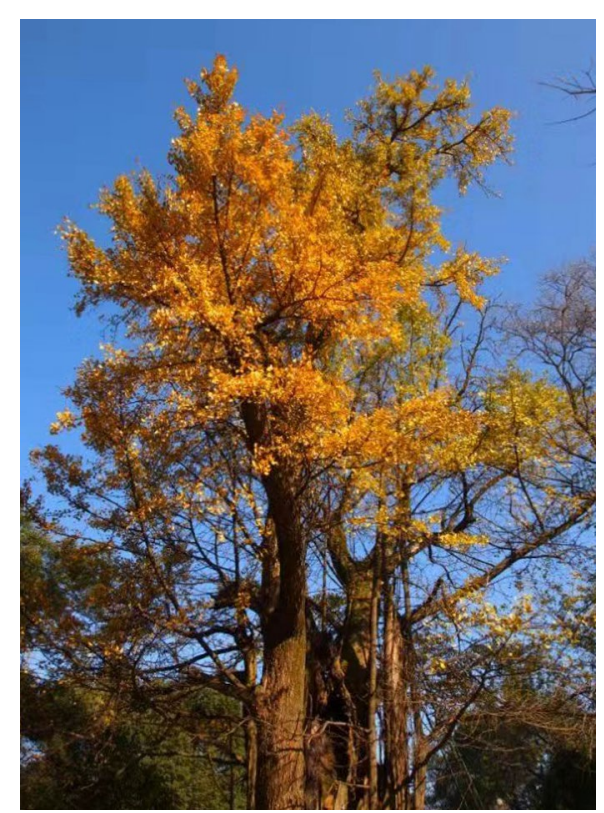

Credit: Image courtesy of Jun Liu

The morphology of the ginkgo tree has been stable for more than 200 million years - one of the outstanding features that make this species an enigmatic living fossil. Ginkgo evolution has long intrigued scientists and has been intensively studied, but it remains unclear how global climate and human activities have shaped this process. A collaborative team from Zhejiang University and the Institute of Botany at the Chinese Academy of Sciences and Beijing Genomic Institute in Qingdao now provides a better understanding by comprehensively analysing the genomes of 545 ginkgo individuals.

The extensive collection of ginkgo individuals was sampled from 51 populations across the world, covering almost all locations where ginkgo grow on the planet. The genome of each individual was sequenced using short-read sequencing, and polymorphic sites were identified across the genome.

The collection can be genetically classified into four major lineages southwestern, southern, eastern and northern, as revealed by population genetic structure and phylogenetic analyses. The deepest split occurred between the southwestern and the eastern plus southern groups, while the northern lineage is admixed with individuals genetically close to the other three lineages. The southwestern, eastern and southern China lineages appeared to be three ancient refugia of ginkgo, and the three relict populations exhibited little gene flow among each other. The high genetic diversity observed suggests that, for living fossils like ginkgo, low morphological variation does not mean low genetic diversity.

The ginkgo species has experienced cycles of population expansions and reductions during the Pleistocene glaciation, likely due to climate fluctuations as shown by demographical history reconstruction. The non-Chinese populations are all genetically close to the eastern lineage, and ginkgo from different continents display differential genetic distances from the eastern lineage, indicating multiple human-mediated introductions of ginkgo from eastern China into North America and Europe.

Finally, the researchers identified the bioclimatic variables that potentially influenced ginkgo distribution and genes that likely contributed to their environmental adaptation, providing further insights into the forces and genetic basis that underlie ginkgo evolution.

\section{Jun Lyu}

Published online: 7 October 2019 https://doi.org/10.1038/s41477-019-0529-2 